\title{
Distributed monitoring system for overhead lines insulations and substations based on wireless capacitive sensors
}

\author{
Damir Zaripov ${ }^{1, *}$, Rustem Nasibullin ${ }^{2}$, and Dinar Zakirov ${ }^{1}$ \\ ${ }^{1}$ Kazan state Power Engineering University, Kazan, Russia \\ ${ }^{2}$ Public Joint Stock Company "Krasnogorsky Zavod", Krasnogorsk, Russia
}

\begin{abstract}
In this paper proposed three modifications to the monitoring system of the overhead lines, insulation state and substations based on sensors installed on supports and insulators. The sensors operate on the principle of capacitive coupling with an insulating structure. Differences between the system modifications in the information present options: light indication for control when bypassing lines and substations and changing graphics on the computer (smartphone) screen when receiving data in real time from sensors using cellular communication. The principle of operation and functional diagrams of the system with results of tests and experiments with manufactured product samples in laboratory and field conditions are presented.
\end{abstract}

\section{Introduction}

Pollution of insulators has historically been one of the main reasons for interruptions in the operation of overhead lines (OHL) and substations (SS) under normal operating conditions. In recent years, the situation with environmental pollution has begun to improve. The main source of pollution affecting equipment today is natural pollution [1]. Today, there is also a significant proportion of outages of overhead lines and substations, which cannot be attributed to pollution by birds or rusty water flowing from the traverse to the insulators. One and the same typical scenario for these outages can be described as they occur on clean or slightly dirty insulators and mostly in the early morning in the presence of dew [2-4]. These faults are generally singlephase with no evidence of pollution during visual inspection following successful automatic reclosing of equipment. The most reason for the frequent shutdowns of overhead lines at night and in the morning has not yet been established. Research is underway.

Continuous monitoring with a distributed sensor system is the most effective way to solve the described problem. Many insulation pollution monitors have been developed over the years [5-10]. Researchers have gone from simple partial discharge (PD) pulse counters to state-of-the-art multichannel devices equipped with integrated weather stations. Using advanced devices, the researchers obtained huge amounts of data, including weather data, which were mainly used to understand the physics of pollution in various types of insulators.

Nowadays, the needs for modern and sophisticated "research" systems for monitoring insulation is decreasing and many companies are switching to systems for simple pollution prevention. They are currently being actively developed. The principle is to cover large areas of data collection and transmission over the internet with these low cost and reliable devices. This paper describes a simple insulation monitoring system based on sensors installed on insulating construction. The goal of the described developments is to create online system for monitoring the of overhead lines insulation and substations.

\section{Construction description}

\subsection{Insulating construction status light}

At the first stage of development, an insulation monitoring system was created and includes a set of sensors with LED indication. The sensors (indicators) have been designed to be mounted on an insulating structure or the end of a portable measuring rod. They signal an increasing of the electric field and the appearance of partial discharges in the area of insulators during inspections of overhead lines and substations. Signals can be observed in the daytime from a distance of up to 30 meters. The main advantages of these sensors: ease of manufacture, low cost and the absence of separate power supplies. The principle behind the operation of the light indicator is based on the capacitive coupling of the sensor and the insulating structure $[11,12]$. The functional diagram of the device is shown in Fig. 1.

\footnotetext{
* Corresponding author: dzaripov@list.ru
} 


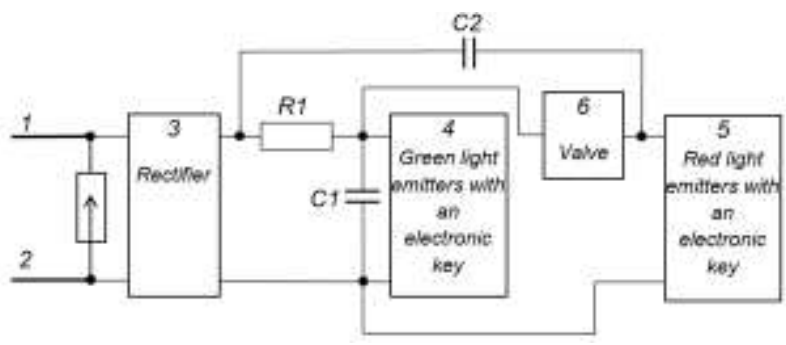

Fig. 1. Functional diagram of the sensor assembly [13].

The device works as follows. A capacitive current flows through the electrode 1, facing the insulating structure, which charges the capacitor $\mathrm{C} 1$ through the rectifier 3 and the resistor $\mathrm{R} 1$. When the insulation is in good condition or the defect is insignificant, there are no partial electrical discharges on the insulation. In this case, when the capacitor $\mathrm{C} 1$ reaches the threshold voltage $(30-50 \mathrm{~V})$ during the charging process, it is discharged through the first light emitter (green) with an electronic key 4 , causing a flash of light. In this case, the voltage across the capacitor drops to a certain lower threshold, the electronic switch stops conducting current and the capacitor $\mathrm{C} 1$ starts charging again, and then the cycle repeats. The repetition rate of green light flashes is periodic and depends on the EMF on the electrode, which, in turn, is determined by the potential distribution along the insulating structure, which changes when the integrity of its individual parts is violated. Elements of the R1 and C2 circuit are selected in such a way that low-frequency oscillations of the network do not penetrate the second light emitter (red) with an electronic key 5 . In addition, due to the voltage drop across valve 6 , the threshold voltage for switching on the second light indicator is always higher and only first indicator light glow.

When the insulation is significantly damaged or contaminated and conditions are created for breakdown or overlap of the insulating structure, the intensity of partial electrical discharges is high. These discharges create pulses of electric current with a duration several orders of magnitude less than the period of the power frequency voltage. At a certain value of these pulses, the voltage at the input of the red-light emitter, due to the low resistance of the capacitor $\mathrm{C} 2$ to high-frequency pulses, can exceed the voltage on the green light emitter, and then it will turn on and will glow first. At high intensity of electrical discharges, only the red emitter will glow. The nature of the glow is not periodic, turning in continuous. Due to the presence of valve 6 , pulses caused by discharges will not affect the operation of the green emitter.

The average degree of insulation damage, when the intensity of electrical discharges is low, will be characterized by the random appearance of flashes of a red-light emitter against a green background. The threshold and frequency of occurrence of red flashes will be determined by the time constant of the capacitor $\mathrm{C} 2$ circuit and this indicator light, which together form a high-frequency filter.

During the experiments on the substation, the light indicator was installed on an insulating rod and brought into contact with the grounded part of the support insulators with one electrode (Fig. 2). On most insulators, the indicator gave uniform pulses with the same frequency green. On separate (11 pieces), random blinking of red LEDs was observed, which indicated a significant intensity of discharges at the surface of the insulation. This assumption was confirmed by measurements with an ultrasonic flaw detector. Maintenance or replacement of insulators recommended.

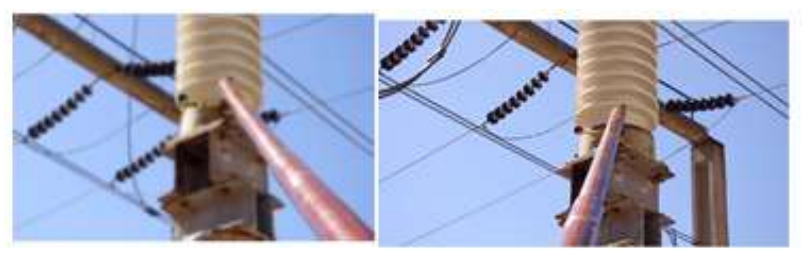

Fig. 2. Indicator on the support insulator in the $110 \mathrm{kV}$ substation (Kazan TES-3).

Real experiments on the overhead network of were carried in Almetyevsk electrical networks. About 50 indicators were randomly installed on $35 \mathrm{kV}$ and $110 \mathrm{kV}$ lines (Fig. 3). During this inspection tour of the $110 \mathrm{kV}$ line section with indicators installed on all phases, an increased pulse repetition rate of the green LED was detected on the polymer insulators of one of the phases of the three supports. This increased EMF on the indicator, caused by a change in the distribution along the insulating structure, was taken for control.

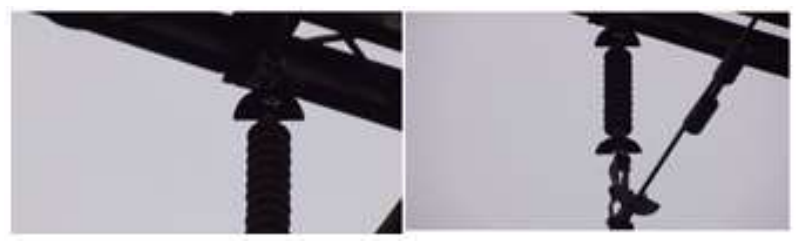

Fig. 3. Indicator on a suspension insulator $35 \mathrm{kV}$ (Almetyevsk electrical networks).

The advantage of the described monitoring system is simple implementation, low cost and reliability. Disadvantage - information is available only through visual control.

\subsection{Light indicator for the insulating construction condition with "memory"}

Insulators with a high probability of the overlap manifest themselves in conditions where there is no possibility of visual observation. Therefore, the above-described sensor circuit with LEDs has been modified in order to save in the device memory the state in which the red LEDs light up, signaling the condition with the appearance of PD. The red LEDs should flash for several days, after which they return to normal operation if the PD disappears. During the development, it was also necessary to preserve an important characteristic of the device - the absence of the need for separate power supplies. To implement this function, a microcontroller (MC) (Fig. 4) [14] is introduced into the above-described sensor (Fig. 4) [14], which stores in memory the fact of 
PD occurrence on the insulator and causes the red LEDs to flash for a user-specified amount of time (about several days). After the specified time has elapsed, the indicator switches to the normal operation.

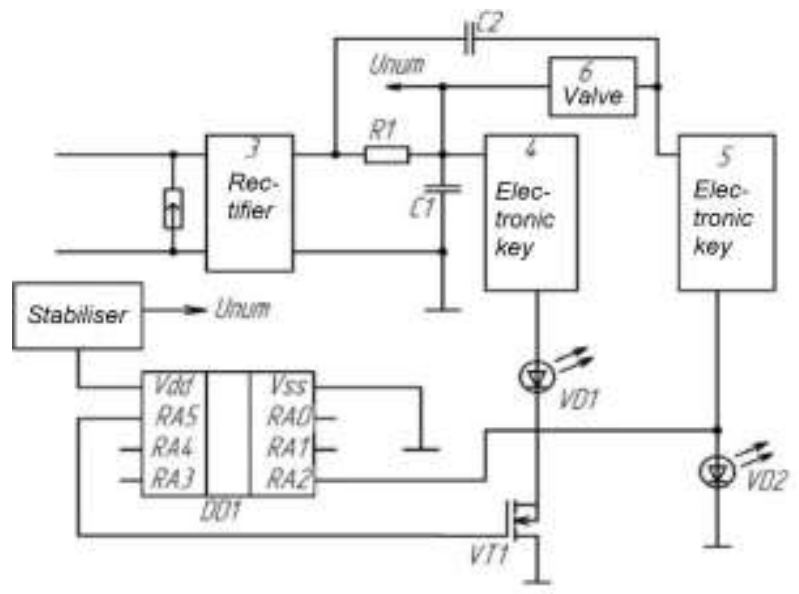

Fig. 4. Sensor diagram with "memory" [14].

The measuring and recording parts work in the same way as the sensor described above in the version with LEDs. When the PD appears, the electronic key of the second signal recorder, the VD2 LED, is triggered. A current pulse passes through the LED, which creates a voltage drop across the LED of about $1.8 \mathrm{~V}$, which corresponds to the logical unit level for the MC. The pulse generated when the PD appears on the indicator is fixed by the microcontroller at the RA2 pin. Next, the MC generates a logical zero signal at the RA5 pin. This signal closes the transistor VT1 for the time set by the microcontroller timer. The closed transistor VT1 does not allow the electronic key of the first signal recorder to operate in the form of the VD1 LED. For this reason, the voltage on $\mathrm{C} 2$ will rise until it reaches the threshold for the electronic key of the second LED. The key of the PD registration channel is triggered, discharging the capacitor $\mathrm{C} 1$ through the second LED. The cycle of charging and discharging $\mathrm{C} 1$ with the ignition of the second LED continues, even if there are no PDs. After the specified blinking time of the second LED, the MC generates a signal to open the transistor VT1. In this case, the electrical circuit of the first LED is closed and the sensor starts to work in normal mode until PD appears again on the insulator. A feature of this scheme, like the previous one, is its autonomy. The PIC12LF1571 microcontroller is selected as a "memory" element. MC is in "sleep mode" most of the time and consumes no more than $10 \mathrm{nA}$ current. The MC current is so small that it can be powered by the charge accumulated on the capacitor $\mathrm{C} 1$, without affecting the operation of the rest of the circuit. The MC is powered by a voltage regulator. The stabilizer uses a floating voltage across the capacitor $\mathrm{C} 1$ as an input voltage. The stabilizer consumes no more than $150 \mathrm{nA}$ current.

According to the described scheme, experimental samples of indicators were made, which were tested with polymer insulators for $110 \mathrm{kV}$ in the high-voltage laboratory of KSPEU. In total, three series of experiments were carried out:
- on a dry insulator;

- on an insulator, soaked in tap water;

- on the insulator, contaminated with brine in a proportion of $20 \mathrm{~g} / \mathrm{l}$ (conductivity $40 \mathrm{mS} / \mathrm{cm}$ for medium pollution).

The insulators were wetted with water and brine by spraying. The change in the period (frequency) of blinking of the LED indicator with "memory" in different series of experiments showed the effect of pollution on the change in the distribution of the electric field along the insulator. The appearance of water drops on an uncontaminated surface practically did not change the field strength in the area of the indicator. At the same time, the saline solution led to the formation of conducting paths with the formation of many intermittent short arcs connecting individual droplets and leading to a noticeable increase in the average field strength in the area of the indicator, as well as the appearance of pulses caused by individual discharges (Fig. 5). In addition, if for wetted surface with water, the red (warning) LEDs did not light up at a voltage significantly exceeding the operating voltage $(65 \mathrm{kV})$, then with a surface contaminated with a saline solution, flashes were observed starting at $60 \mathrm{kV}$. In accordance with the developed circuit, because of the memory, the glow of the red LEDs was preserved even if the voltage was significantly lower than the operating voltage. The advantage of this version of the distributed monitoring system of insulators is the ability to detect hidden defects and contaminants that appear when the insulator is wetted.

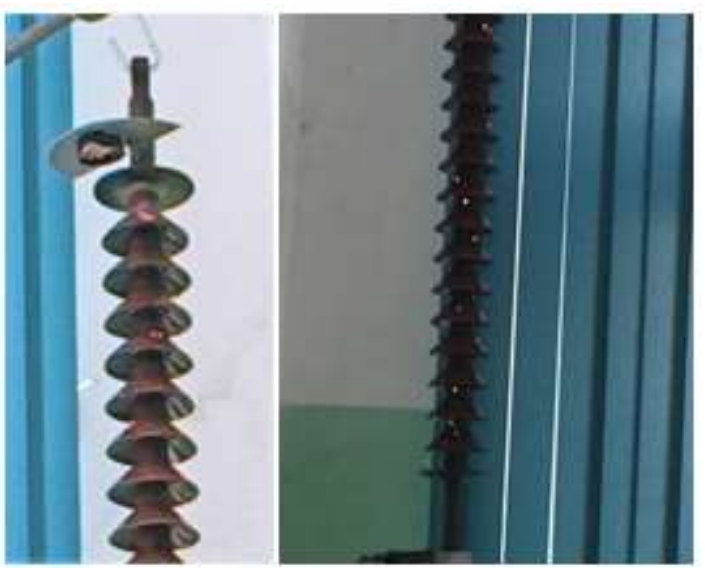

Fig. 5. The picture of the discharge activity on the insulator with pollution in the laboratory [14].

\subsection{Insulation monitoring system for overhead lines with continuous data transmission using a wireless network}

For continuous monitoring of the insulators condition, a wireless system was developed, which, comparing other devices described above, transmits the signals to a computer (smartphone) generated by the sensor in real time [11]. The system contains sensors, hubs and mobile computers that receive and process data in real time (Fig. 6). 


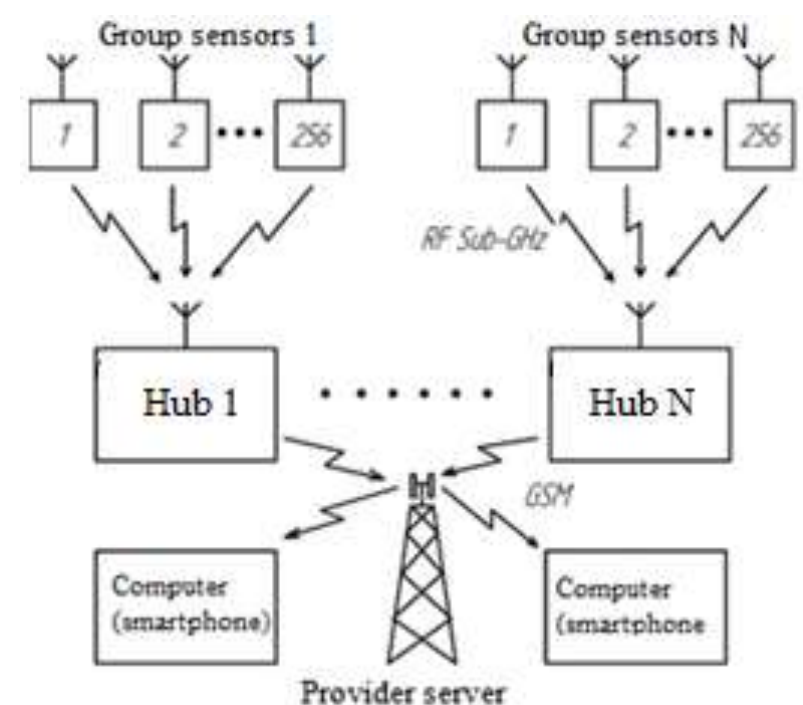

Fig. 6. Insulation monitoring system for overhead lines with cellular data transmission.

Sensors installed on insulating constructure transmit data at a predetermined time interval, and a concentrator installed nearby, receiving data from sensors, transmits it through GSM radio channel to services department. The software for smartphone or personal computer receives data of the insulators condition and visualizes them in the form of changing graphs in the electric field strength in the area of the insulating structure, the presence and intensity of discharges, and also signals an accident when complete overlap or breakdown of insulators. The analysis of the graphs allows predicting the failure for insulators and planning preventive measures. The sensor consists of an electrode (El), a rectifier, a pulse shaper unit (PSU), a microcontroller (MC), a wireless transmitter (WT) and a power supply (PS) (Fig. 7). All nodes are located on one printed circuit board, including the electrode, which is made in the form of a metallization layer (Fig. 8 a).

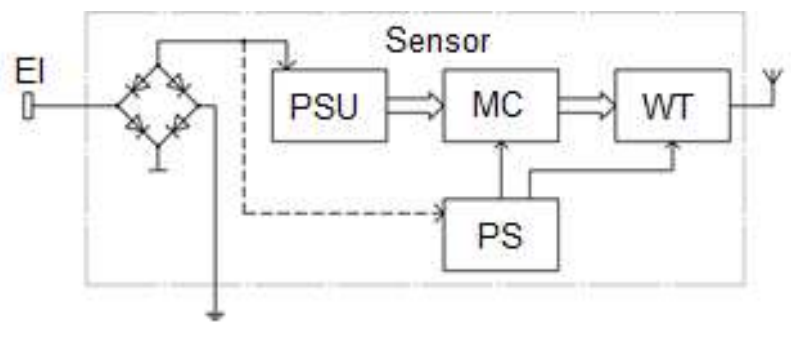

Fig. 7. Functional diagram of the sensor.

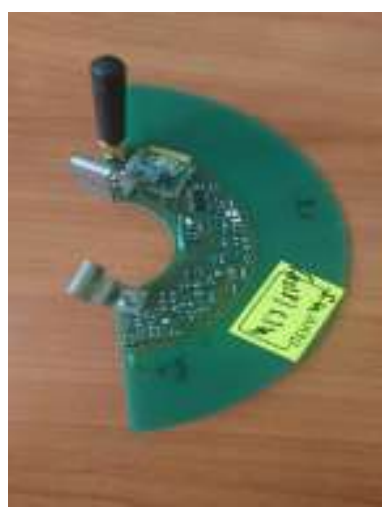

a)

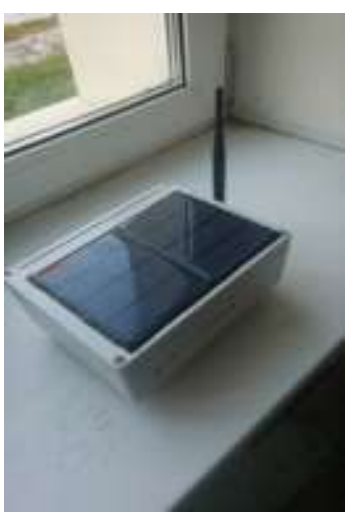

b)
Fig. 8. Experimental samples of the sensor (a) and the concentrator (b).

The electrode, which performs the function of the capacitor lining, forms a capacitor together with the high-voltage wire of the overhead line. It is connected to the overhead line supporting structure (to "ground") through the measuring part of the sensor. When an alternating electric field appears on the power line, a current begins to flow through the sensor electrode, which, passing through the sensor, is rectified and converted into electrical impulses. The pulse frequency is proportional to the amount of current.

The pulse shaping unit has 2 channels. It is made on 2 DB4 dinistors, which opens when their outputs reach the trigger voltage (about $35 \mathrm{~V}$ ). One dinistor works in the low-frequency signal circuit and generates pulses with a frequency proportional to the electric field strength in the area of the sensor. The second dinistor operates in a high-frequency signal circuit caused by partial discharges near the insulator. This dinistor opens when the partial discharge voltage pulse reaches the threshold value. Thus, the first measurement channel generates pulses, the frequency of which is proportional to the electric field strength (or the averaged leakage current), and the second measurement channel counts the number of discharge pulses exceeding the threshold value.

To receive data over a radio channel from sensors located at a distance of up to $1 \mathrm{~km}$, and then transmit them to a smartphone with a given frequency through cellular communication, a device (hub) with two unidirectional data transmission channels was designed (Fig. 8 (b)). Channel 1 receives data from sensors. Channel 2 transfers the collected data to the remote computer or smartphone. The central component of the system is a microcontroller. Its main function is to exchange information with other components, as well as to process information in accordance with the algorithms embedded in its program. The hardware chosen was the Arduino Mega 2560 based on the ATmega2560 microcontroller. The advantage of the Arduino Mega, like other Arduino models, it's easy to writing a program in a special development environment Arduino IDE and further "firmware" without using a separate programmer. Another important advantage of the Arduino Mega is the availability of hardware-supported SPI and UART communication interfaces, which are necessary for 
exchanging information with other devices. The RFM69HW-868-S2 transceiver was chosen to receive data from the sensors. Its advantages are high sensitivity, low power consumption and a library of functions for the Arduino IDE, which makes it easy to work with.

To transfer data into smartphone through the cellular network, you need a GSM/GPRS data transmission module that is compatible with SIM cards. For these purposes we chose the SIM800L module. Its advantages includes low power consumption in idle mode and the ability to put it into "sleep mode" with even lower power consumption. For autonomous operation of the concentrator, four Li-Ion batteries with a nominal voltage of $3.2 \mathrm{~V}$ are used. They have a capacity of about $2000 \mathrm{mAh}$, which, with optimal power consumption of the system, will be sufficient for long-term operation. The batteries are recharged by two solar panels mounted on the concentrator cover. Tests have shown that four hours in the sun after two days without the sun is enough to recharge the batteries. The test of the operation of the experimental sample of the insulation condition monitoring system was carried out in the high-voltage laboratory of the KSPEU. The sensors were installed around the LK 70/110 polymer insulator at random, as shown, for example, in Fig. 9.

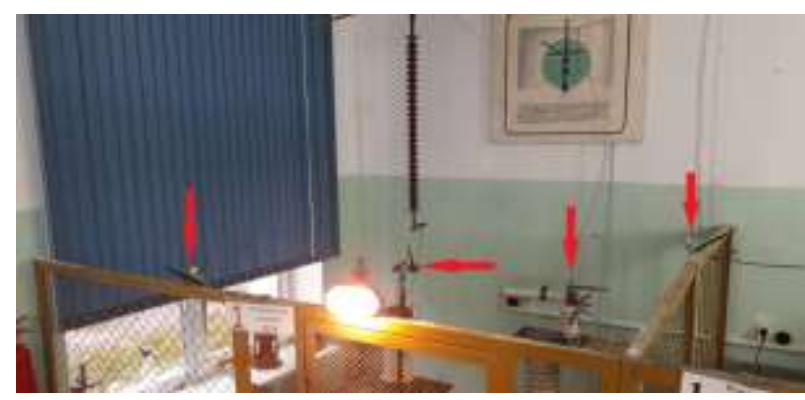

Fig. 9. Insulator LK 70/110 with sensors installed around in the laboratory.

A high voltage was applied to the insulator and, at an interval of 1 minute, the data received from the sensors was recorded in smartphone. The high-voltage was increased discretely from $40 \mathrm{kV}$ to $80 \mathrm{kV}$ (before the appearance of noticeable PDs) with a step of $10 \mathrm{kV}$ per minute. On the smartphone screen (Fig. 10), the incoming data was displayed in the form of three graphs:

- blue (low-frequency channel, voltage change on the sensor when the alternating voltage on the insulator changes);

- red (high-frequency channel, the appearance and growth of PD);

- orange (the channel displaying a short circuit (SC) was not tested in the experiment, the value on the graphs is always 0 ).

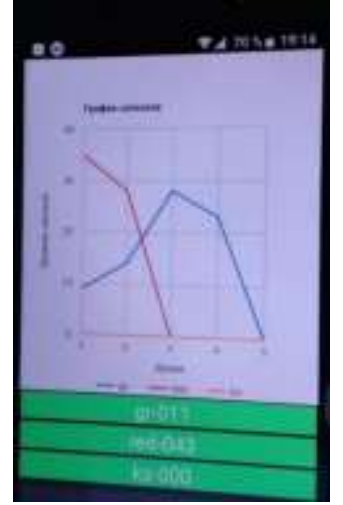

Fig. 10. Graphs of signal changes on the smartphone screen (screenshot of the smartphone).

An experimental batch of several sensors and concentrators was installed on separate supports of 110 and $220 \mathrm{kV}$ overhead lines of Almetyevsk electrical networks and passed the first tests. The system showed its performance as intended during these tests, and despite the identified design flaws, a number of interesting results were obtained. During the operation of the sensors for several months in the summer and autumn period, signals from sensors about insignificant discharge activity on polymer insulators were repeatedly received on the smartphone. This mainly happened in bad weather. The situation that occurred at the end of the August and the beginning of September 2019 stands out from this series, when on one of the sensors located on the side of the polymer insulator, signals of intense PD appeared for three days, which appeared early in the morning and subsided by the middle of the day. The weather during this period was sunny. Presumably this activity was due to fog.

\section{Conclusion}

In the presented work, based on the analysis of the world experience in the operation of insulators on overhead lines, the relevance of the development of insulation monitoring systems for overhead lines with a distributed installation on supports or insulators of continuously operating sensors is shown. The developed options allow you to choose the monitoring method: visual, if it is possible for personnel to bypass the lines, or online, when monitoring hard-to-reach, contaminated sections of lines with frequent outages. Despite the difficulties of today with the introduction of this technology, associated with the reliability and autonomy of the operation of sensors, with their installation at the facility, etc., a further increase in the reliability of overhead lines is seen in the transition to such systems.

The research was carried out with the financial support of the Ministry of Science and Higher Education of the Russian Federation within the framework of the state assignment for the implementation of research work on the topic "Distributed automated systems for monitoring and diagnosing the technical condition of overhead power lines and substations based on the technology of broadband data transmission through 
power lines and the industrial Internet of things" (no. topics 075-00063-20-02).

\section{References}

1. R. Arbuzov, A. Ovsyannikov, News of electrical engineering, 4 (2008)

2. D. Hoch, N. Mahatho, F. Bolonga, Electronic resource (2006) Available at: https://www.researchgate.net/publication/255570 809_WATER_INDUCED_DISCHARGES_ON_ TRANSMISSION_VOLTAGE_SILICONE_RU BBER_INSULATORS

3. V.G. Borovitsky, Study of the causes of unmotivated shutdowns of $110 \mathrm{kV}$ overhead lines and the development of recommendations for reducing their number: Dis. Cand. Tech. Sciences (Novosibirsk, 201, 2013)

4. Research Provided Insight into Unexplained Line Outages [Electronic resource] (2019) Available at: http://www.inmr.com/research-providedinsight-unexplained-line-outages/

5. A. Levinzon, D. Kottick, R. Knijnik, L. Frenkel, B2-205, CIGRE (2012)

6. A. Phillips, D. Mcguire, C. Engelbrecht, B3-301, CIGRE (2016)

7. X. Huang, Q. Sun, J. Ding, 3rd IEEE Conference on Industrial Electronics and Applications, Singapore, pp. 891-896 (2008)

8. A. Phillips, F. Bologna, J. Major, C. Engelbrecht, Proceedings of the 16th International Symposium on High Voltage Engineering, Cape Town, South Africa, 5-3, 1-6

9. S. Campello Oliveira, E. Fontana, R. Bandeira Lima, IEEE Transactions Power Delivery, 24 (4), 2257-2260 (2009)

10. V.V. Startsev, V.A. Lyubimov, E.P. Soloviev, Yu.A. Solodkov, High voltage insulation status indicator, RF pat. 2392679, priority of 20 June 2010, (2012)

11. D.K. Zaripov, R.A. Nasibullin, R.R. Ibragimov, M.Ch. Igtisamov, Sensor for insulating structures condition continuous monitoring system, RF Pat. 2731169, priority of 30 December 2019 (2020)

12. D.K. Zaripov, Optical remote diagnostics method for insulating structure, RF Pat. 2517776, priority of 03 December 2012 (2014)

13. D.K. Zaripov, R.A. Nasibullin, S.M. Margulis, R.R. Ibragimov, M.Ch. Igtisamov, Light indicator of the state of the insulating structure state, RF Pat. 2660754, priority of 14 July 2017 (2018)

14. D.K. Zaripov, R.A. Nasibullin, Kontenant, 17 (4), 80-90 (2018) 